\title{
In vitro release and in vitro-in vivo correlation for silybin meglumine incorporated into hollow-type mesoporous silica nanoparticles
}

This article was published in the following Dove Press journal:

International Journal of Nanomedicine

17 February 2012

Number of times this article has been viewed

\author{
Xia Cao* \\ Wen-Wen Deng* \\ Min $\mathrm{Fu}^{*}$ \\ Liang Wang \\ Shan-Shan Tong \\ Ya-Wei Wei \\ Ying $\mathrm{Xu}$ \\ Wei-Yan Su \\ $\mathrm{Xi}$-Ming $\mathrm{Xu}$ \\ Jiang-Nan Yu \\ Department of Pharmaceutics, \\ School of Pharmacy, and Center \\ for Nano Drug/Gene Delivery and \\ Tissue Engineering, Jiangsu University, \\ Zhenjiang, People's Republic of China \\ *These authors contributed equally \\ to this work
}

Correspondence: Xi-Ming Xu; Jiang-Nan Yu School of Pharmacy, Jiangsu University, 30I Xuefu Road, Jingkou District,

Zhenjiang 21200I,

People's Republic of China

$\mathrm{Tel}+865$ II 8503845 I

Fax +865 II 8503845 I

Email xmxu@ujs.edu.cn;yjn@ujs.edu.cn
Background: The purpose of this study was to develop a sustained drug-release model for water-soluble drugs using silica nanoparticles.

Methods: Hollow-type mesoporous silica nanoparticles (HMSNs) were prepared using $\mathrm{Na}_{2} \mathrm{CO}_{3}$ solution as the dissolution medium for the first time. The water-soluble compound, silybin meglumine, was used as the model drug. The Wagner-Nelson method was used to calculate the in vivo absorption fraction.

Results: The results of transmission electron microscopy and nitrogen adsorption revealed that the empty HMSNs had uniformly distributed particles of size 50-100 nm, a spherical appearance, a large specific surface area $\left(385.89 \pm 1.12 \mathrm{~m}^{2} / \mathrm{g}\right)$, and ultralow mean pore size $(2.74 \mathrm{~nm})$. The highly porous structure allowed a large drug-loading rate $(58.91 \% \pm 0.39 \%)$. In $0.08 \mathrm{M} \mathrm{Na}_{2} \mathrm{CO}_{3}$ solution, silybin meglumine-loaded HMSNs could achieve highly efficacious and long-term sustained release for 72 hours in vitro. The results of in vitro-in vivo correlation revealed that HMSNs in $0.08 \mathrm{M} \mathrm{Na}_{2} \mathrm{CO}_{3}$ solution had a correlation coefficient $\mathrm{R}^{2}$ value of 0.9931 , while those of artificial gastric juice and artificial intestinal juice were only 0.9287 and 0.7689 , respectively.

Conclusion: The findings of in vitro-in vivo correlation indicate that HMSNs together with $\mathrm{Na}_{2} \mathrm{CO}_{3}$ solution could achieve an excellent linear relationship between in vitro dissolution and in vivo absorption for 72 hours, leading to a promising model for sustained release of watersoluble drugs.

Keywords: hollow-type mesoporous silica nanoparticle, silybin meglumine, in vitro dissolution, in vivo absorption, in vitro-in vivo correlation

\section{Introduction}

Over the past few decades, mesoporous silica nanoparticles have played an important role in the development of drug delivery systems. They have made a significant impact on medical technology by minimizing the size of drug delivery devices from the macro to the nano scale and significantly enhancing the performance of existing lifesaving drugs. ${ }^{1,2}$ Mesoporous silica nanoparticles have attracted considerable attention due to their ability to enhance solubility of poorly water-soluble drugs, as well as to achieve sustained drug release, leading to improved bioavailability. ${ }^{3,4}$ Advantages of mesoporous silica nanoparticles, such as large surface area, high pore volume, tunable pore size, and well defined pore structure, as well as mechanical and chemical stability and protection of the integrity of the molecular structure of the drug, ${ }^{5-7}$ make them ideal for controlled drug delivery systems. Most recently, mesoporous silica nanoparticles were developed for intracellular controlled drug/gene delivery ${ }^{8,9}$ and targeted drug delivery to cancer cells. ${ }^{10,11}$ Furthermore, the safety of silica materials 
used as drug delivery systems has been well defined in several reports, ${ }^{12,13}$ which makes them desirable as perfect delivery systems. Chervenkov et $\mathrm{al}^{12}$ found that oral $(2 \mathrm{~g} / \mathrm{kg}$ bodyweight) administration of silica dioxide xerogels did not result in significant changes in the biochemical parameters characterizing liver function and gastric mucosal damage.

So far, many attempts have been made to develop sustained-release preparations to improve the release behavior of orally delivered water-soluble drugs, such as sustainedrelease matrix tablets, ${ }^{14,15}$ nanostructured liquid crystalline matrix, ${ }^{16}$ surfactant-polymer nanoparticles, ${ }^{17}$ double-walled microspheres, ${ }^{18}$ and mesoporous silica nanoparticles. ${ }^{3,5}$ However, the in vitro sustained-release duration of these formulations lasted no longer than 24 hours. Therefore, it is desirable to develop a drug-release model that can achieve in vitro sustained release for a long period of time. In the present study, hollow-type mesoporous silica nanoparticles (HMSNs) were prepared and used as an in vitro sustained drug-release model for water-soluble drugs. Due to the highly porous structure of HMSNs, drugs can be loaded within the pores or canals, achieving in vitro sustained release for 72 hours. HMSNs have most of the advantages of mesoporous silica nanoparticles, but unlike mesoporous silica nanoparticles published earlier on, HMSNs possess a spherical shape with inner pores and channels and longer-lasting drug release in vitro. The possible reason is that, unlike the regularly and orderly distributed canals in mesoporous silica nanoparticles, the HMSNs prepared in this study possessed irregular and disordered canals which may result in prolonged drug release because it is harder for the drug to escape from disordered canals than from ordered ones.

Silybin meglumine, a commercially used compound of silybin and meglumine, was used as the model drug in this work. It has been widely used for the treatment of acute hepatitis, chronic hepatitis, initial-stage hepatocirrhosis, and liver damage due to intoxication because of the excellent hepatoprotective effect of silybin. Silybin is a naturally occurring polyphenolic flavonoid extracted from the seed of the milk thistle (Silybum marianum), ${ }^{19,20}$ and has been widely used to treat certain liver disorders, such as chronic active hepatitis, hepatic cirrhosis, as well as alcohol-induced and various types of drug-induced and toxin-induced liver damage. ${ }^{21,22}$ It is known that silybin is a poorly water-soluble drug; however, once silybin is combined with meglumine, its ionization ability increases and silybin meglumine is endowed with high water solubility. The present commercial formulation of silybin meglumine is in the form of a tablet and patients have to take the medicine three times a day in order to maintain an effective plasma concentration. Obviously, it is inconvenient for most patients to take medicine so frequently. Therefore, it is important to develop a universally applicable technology for processing silybin meglumine in order to achieve high-efficacy, a long-term drug-release pattern, and reduce the frequency of drug administration. Furthermore, such a delivery system must be safe after oral or intravenous administration.

Until now, most investigations of silica nanoparticles have focused on porous silica nanoparticles and mesoporous silica nanoparticles, including preparation methods, drug-loading capacity, and in vitro release performance. ${ }^{23-25}$ Few attempts have been made to evaluate their in vivo absorption characteristics, and no research has been carried out to study in vitro-in vivo correlations (IVIVCs). Further, the present scientific literature shows that no report has been published about HMSNs for sustained drug delivery. In a report by Li et al, ${ }^{26}$ the in vitro controlled release of loaded drugs from porous hollow silica nanoparticles could last for up to 30 days, but no in vivo investigation was carried out. Based on common knowledge, loaded drugs cannot survive up to 30 days after release in vivo. Previous studies have confirmed that the in vitro accumulated release of drug-loaded HMSNs in traditional dissolution media was less than $20 \%$, but the drug could be eliminated within 72 hours following single-dose administration in Beagle dogs (data not shown). This inconsistency between in vitro dissolution and in vivo absorption has prompted researchers to seek out improved dissolution methods or better dissolution media in order to obtain better IVIVCs.

The in vitro dissolution medium is an important factor in constructing a good in vitro drug-release model. So far, many media, such as phosphate buffer solution, ${ }^{27} \mathrm{HCl}$ solution (pH 1-2), ${ }^{4}$ and water/ethanol mixture (70:30, v/v), ${ }^{26}$ have been used to dissolve formulations prepared on the basis of porous silica nanoparticles, but none of these studies has evaluated IVIVCs. A possible reason is that there still remain many difficulties in developing appropriate dissolution test methods due to fast dissolution of submicron drug substances and in separating undissolved particles from dissolution medium. ${ }^{28}$ What is more important is to find a proper dissolution medium that can achieve a good IVIVC.

In an effort to address the problems mentioned above, our research group attempted to develop a high-efficacy, long-acting in vitro drug-release model based on HMSNs for the delivery of water-soluble drugs. Water-soluble silybin meglumine was directly loaded into HMSNs in this study. The in vitro release profiles of silybin meglumine-loaded HMSNs were studied for the first time using $\mathrm{Na}_{2} \mathrm{CO}_{3}$ solutions with different concentrations $(0.01 \mathrm{M}, 0.06 \mathrm{M}, 0.08 \mathrm{M}$, and $0.1 \mathrm{M})$. The treatment was repeated for other dissolution media such as traditional 
artificial gastric juice ( $\mathrm{pH}$ 1.2) and artificial intestinal juice (pH 6.8). Beagle dogs were used as an in vivo model to study pharmacokinetic characteristics after oral administration, and the IVIVC was then established. The use of HMSNs together with a $\mathrm{Na}_{2} \mathrm{CO}_{3}$ solution at a certain concentration is expected to be developed into an effective approach to obtain an in vitro drug-release model with a sustained-release pattern lasting for 72 hours, as well as a good IVIVC.

\section{Methods and materials Materials}

Tetraethyl orthosilicate, absolute ethyl alcohol, ammonia water, cyclohexane, n-hexanol, and $\mathrm{Na}_{2} \mathrm{CO}_{3}$ were purchased from Chemical Reagent Co, Ltd, of the China National Pharmaceutical Group (Shanghai, China). NP-10 was obtained from Shanghai Jiafang Trade Co, Ltd (Shanghai, China). The animal experiments were performed strictly according to the experimental protocols approved by the university ethics committee for the use of experimental animals, and also conformed to the Guide for Care and Use of Laboratory Animals.

\section{Preparation of empty HMSNs}

A $6 \mathrm{~mL}$ quantity of nonylphenol 10 (NP-10) was added to $50 \mathrm{~mL}$ of cyclohexane, and shaken together. After adding $2.2 \mathrm{~mL}$ of $\mathrm{n}$-hexanol and $1.8 \mathrm{~mL}$ of $25.6 \%$ ammonia water, the resulting mixture was again agitated for one hour at room temperature. Tetraethyl orthosilicate $4.2 \mathrm{~mL}$ was slowly added to the mixture and agitation was conducted for another 24 hours at room temperature. A $60 \mathrm{~mL}$ quantity of absolute ethyl alcohol was then added prior to ultrasound treatment for one hour (Wuxi Ultrasonic Devices Factory, Jiangsu, China). Centrifugal separation was performed at 15,000 per second for 15 minutes and the precipitate was washed three times with double-distilled water. After addition of water, freezing, and drying of the substance in succession, a powder of solid silica nanoparticles was obtained.

After addition of $1 \mathrm{~g}$ of the silica nanoparticles to $1000 \mathrm{~mL}$ of $0.6 \mathrm{~mol} / \mathrm{L} \mathrm{Na}_{2} \mathrm{CO}_{3}$ solution, ultrasound was administered for 5 minutes at $65^{\circ} \mathrm{C}$ and $200 \mathrm{~W}$. The mixture was then centrifugally separated at 15,000 per second for 15 minutes and the precipitate was washed three times with double-distilled water. The resulting precipitate was then added to $10 \mathrm{~mL}$ of double-distilled water, followed by freezing and drying the substance in succession, and empty HMSNs were thus obtained. Fourier transform infrared analysis was carried out to examine the residual surfactant (NP-10). No absorption band characterizing NP-10 was observed, indicating that the surfactant had been completely washed away.

\section{Preparation of silybin meglumine-loaded HMSNs}

In this study, silybin meglumine was directly loaded into the HMSNs. Two grams of silybin meglumine was dissolved in $20 \mathrm{~mL}$ of double-distilled water, in which $1 \mathrm{~g}$ of empty HMSNs were soaked for 24 hours and then centrifugally separated at 15,000 per second for 15 minutes. The precipitate was washed three times with double-distilled water. It was then added to $10 \mathrm{~mL}$ of double-distilled water, then frozen and dried in succession. Drug-loaded nanoparticles were obtained.

\section{Physicochemical characterization of HMSNs}

The physicochemical features of HMSNs were characterized using transmission electron microscopy and the nitrogen adsorption method. The morphology and internal porous structure of the samples were observed using a transmission electron microscope (JEM-2100, JEOL, Tokyo, Japan). The specific surface area, pore volume, and pore diameter of the samples were measured by the nitrogen adsorption method using a high-speed automated surface area and pore size analyzer (Nova 2000, Quantachrome Instruments, Boynton Beach, FL) according to the Brunauer-Emmett-Teller and Barrett-Joyner-Halenda procedures.

The drug-loading rates of both silybin meglumine-loaded HMSNs and silybin meglumine-loaded solid silica nanoparticles were also determined. A $10 \mathrm{mg}$ quantity of silybin meglumine-loaded HMSNs and the same amount of silybin meglumine-loaded solid silica nanoparticles were added to a certain amount of $\mathrm{Na}_{2} \mathrm{CO}_{3}$ solution and then water-bathed at a certain temperature for 30 minutes. The treatment was repeated for the same amount of HMSNs without a loading drug which resulted in a blank control solution.

The drug-loading rate was determined via ultraviolet spectroscopy (UV-2401PC, Shimadzu, Tokyo, Japan) at a wavelength of $288 \mathrm{~nm}$. The blank control solution was used as reference. The drug-loading rate was calculated according to the following formula:

Drug loading rate $=\frac{\text { Weight of drug in HSNs or SSNS }}{\text { Weight of drug loaded HSNs or SSNs }} \times 100 \%$

\section{In vitro sustained-release studies}

The in vitro release profiles of silybin meglumine from the sample were determined by a dialysis tubing method using dissolution testing apparatus (Tianjin University Radio Factory, Tianjin, China) according to the paddle method recorded by the Chinese Pharmacopoeia (2005). Artificial 
gastric juice ( $\mathrm{pH}$ 1.2), artificial intestinal juice $(\mathrm{pH} 6.8)$, $\mathrm{Na}_{2} \mathrm{CO}_{3}$ solutions with different concentrations $(0.01 \mathrm{M}$ pH 11.1, 0.06 M pH 11.5, 0.08 M pH 11.6, 0.1 M pH 11.7), and $0.01 \mathrm{M} \mathrm{NaOH}(\mathrm{pH} 12)$ were used as dissolution media in this study. The paddles were rotated at $100 \mathrm{rpm}$ for 72 hours. The samples were accurately weighed and added to the dialysis bags, which were then fixed on the paddles. The samples were then put into $900 \mathrm{~mL}$ of artificial gastric juice (pH 1.2) or intestinal juice $(\mathrm{pH} 6.8)$ as the dissolution medium with the temperature adjusted to $37^{\circ} \mathrm{C} \pm 0.1^{\circ} \mathrm{C}$.

Test fluids were withdrawn at predetermined time intervals from each vessel and then filtered through a $0.45 \mu \mathrm{m}$ membrane filter. The same volume of test fluid removed was then replaced by fresh medium. The quantity of silybin in the filtrate $(20 \mu \mathrm{L})$ was analyzed using a high-pressure liquid chromatography (HPLC) system (model 510; Waters Corporation, Milford, MA) equipped with an ultraviolet detector (model 486; Waters Corporation). The HPLC conditions were as follows: reverse-phase column, Nova-pack C18 column $(3.9 \times 150 \mathrm{~mm}, 5 \mu \mathrm{m})$ at $37^{\circ} \mathrm{C}$; mobile phase, dipotassium hydrogen phosphate $(0.05 \mathrm{M})$ : methanol 9.9:11 (v/v) mixture; flow rate, $1.0 \mathrm{~mL} / \mathrm{minute}$; and detection wavelength, $288 \mathrm{~nm}$. All analyses were performed at $37^{\circ} \mathrm{C}$ and experiments were performed at least three times. The standard curve range was $1.68-31.8 \mu \mathrm{g} / \mathrm{mL}$.

\section{In vivo absorption study}

\section{Animal experiment}

Healthy male Beagle dogs weighing $10 \pm 2 \mathrm{~kg}$ were subjected to fasting overnight before the experiments, but allowed free access to water throughout the study. Silybin meglumine-loaded HMSNs were administered as an oral dose $(21.2 \mathrm{mg} / \mathrm{kg})$ to the test group of six dogs.

Serial blood samples of approximately $3 \mathrm{~mL}$ were collected at $0.25,0.5,1,2,4,6,8,10,12,16,20,24,36,48,60$, and 72 hours after oral administration. Plasma obtained after centrifugation (10 minutes, 4000 per second) was immediately added to $20 \mu \mathrm{L}$ of internal standard solution ( $\alpha$-naphthol, $10 \mu \mathrm{g} / \mathrm{mL}$ in methanol) followed by gentle vortex agitation for 5 minutes. The mixture was centrifuged for 10 minutes at 3000 per second, and the organic layer was then transferred to a clean vial and evaporated under nitrogen at $30^{\circ} \mathrm{C}$. Finally, the residue was dissolved in $100 \mu \mathrm{L}$ of the mobile phase and transferred to the HPLC system for analysis.

\section{HPLC analysis}

Plasma silybin was determined by HPLC. A Nova-pack C18 column $(150 \mathrm{~mm} \times 3.9 \mathrm{~mm}, 5 \mu \mathrm{m})$ with a column temperature of $37.5^{\circ} \mathrm{C}$ and a mobile phase consisting of methanol and $\mathrm{KH}_{2} \mathrm{PO}_{4}(0.05 \mathrm{~mol} / \mathrm{L})$ with the ratio of $9.9: 11$ at $\mathrm{pH} 3.8$ were used. The eluent was monitored at $288 \mathrm{~nm}$ with a flow rate of $1 \mathrm{~mL} /$ minute. $\alpha$-Naphthol was used as an internal standard.

\section{Calculation of in vivo absorption percentage}

There are various methods that can be used to calculate the in vivo absorption percentage, including Wagner-Nelson and Loo-Riegelman methods. The former is applicable to a one-compartment model, the later is suitable for a two-compartment model, whereas according to the result determined by Akaike's information criterion method, which was conducted by using software BAPP2.3 (supplied by the Center of Drug Metabolism of China Pharmaceutical University, China) in our previous studies, the plasma concentration-time data for free silybin released from HMSNs were fit to a one-compartment model (data not shown). Therefore, the Wagner-Nelson method was used in the present work to calculate the in vivo absorption fraction:

$$
f=\frac{C t+k \int_{0}^{T} C t d t}{k \int_{0}^{\infty} C t d t} \times 100 \%
$$

In the above formula, $f$ represents the in vivo absorption fraction, $C t$ is the plasma concentration at time point $t$, and $k$ is the elimination rate constant.

\section{Statistical analysis}

All values are represented as the mean \pm standard deviation. Statistical differences between the different groups were determined using the Student's $t$-test and one-way analysis of variance, with a least significant difference post-hoc test by SPSS statistical software (SPSS version 15.0, SPSS Inc, Chicago, IL). Differences between the two groups were considered to be statistically significant at $P<0.01$.

\section{Results and discussion Preparation and physicochemical characteristics of HMSNs}

So far, a number of studies have been conducted on the preparation of porous silica spheres by the modified Stober methods, in which cationic surfactants were used as porogens. ${ }^{29-31}$ In the present study, a $\mathrm{Na}_{2} \mathrm{CO}_{3}$ solution and 
ultrasound were used to make pores and canals within the silica spheres. After lyophilization, the hollow-type mesoporous silica nanoparticles were then obtained. In this work, $\mathrm{Na}_{2} \mathrm{CO}_{3}$ solution was used innovatively to dissolve the silica partially under certain conditions. The resulting HMSNs were highly porous with a uniform small size, and had disordered holes, as well as a large surface area.

Transmission electron microscopy was used to characterize the morphology and porous structure of the HMSNs. It was observed that the empty/drug-loaded HMSNs with/ without phosphotungstic acid staining, showed images of empty/drug-loaded HMSNs (with phosphotungstic acid staining) being less desirable as compared with the other component without phosphotungstic acid staining (data not shown). Thus, the transmission electron microscopy without phosphotungstic acid staining was used in the present study. Figure 1 shows transmission electron microscopic images of solid silica nanoparticles and HMSNs before and after drug-loading, in which a spherical appearance and uniformly distributed particle size within the range of 50-100 nm were observed. In addition, the highly porous inner structure of the empty HMSNs (Figure 1B) was clearly visible in comparison with the solid silica nanoparticles (Figure 1A). When loaded with drugs, the holes and canals on the surface of the particles disappeared, as shown in Figure 1C (silybin meglumine-loaded HMSNs). The most probable reason to explain this observation is that most of the canals and holes within the empty HMSNs were filled with drug.

In this study, to identify the specific surface area, pore volume, and pore diameter of the samples, the nitrogen adsorption method was employed. The results are shown in Figure 2, in which the nitrogen adsorption-desorption isotherms of both solid silica nanoparticles and HMSNs before and after drug-loading are demonstrated. The nitrogen adsorption-desorption isotherms for solid silica nanoparticles completely overlapped, indicating no presence of pores, while an adsorption isotherm of empty HMSNs similar to Type II Langmuir adsorption isotherms and a hysteresis loop in the range of relative pressure from 0.696 to 0.989 indicated the presence of pores or canals in HMSNs. ${ }^{32}$ The average pore diameter of the empty HMSNs was $2.74 \mathrm{~nm}$ and the pore size was uniformly distributed within a relatively narrow range, between $1 \mathrm{~nm}$ and $6 \mathrm{~nm}$. The empty HMSNs with an ultranarrow pore size ( $2.74 \mathrm{~nm}$ on average) prepared in this work were obviously smaller than that of the porous silica nanoparticles reported by Zhang et al. ${ }^{3}$ When loaded with drug, HMSNs showed no hysteresis loop, suggesting that most pores within the HMSNs were completely filled with drug. These results are in agreement with the transmission electron microscopic image in Figure 1. Additionally, nitrogen adsorption results demonstrated that the HMSNs had a much larger specific surface area $\left(385.89 \pm 1.12 \mathrm{~m}^{2} / \mathrm{g}\right)$ than the solid silica nanoparticles $\left(5.84 \pm 0.26 \mathrm{~m}^{2} / \mathrm{g}\right)$, indicating the highly porous structure of HMSNs. After adsorption of silybin meglumine, a significant decrease was observed in the surface area of HMSNs $\left(6.42 \mathrm{~m}^{2} / \mathrm{g}\right)$ and the pore diameter $(1.68 \mathrm{~nm})$, indicating successful loading of silybin meglumine.

The drug-loading rate of the silybin meglumine-loaded HMSNs was $58.91 \% \pm 0.39 \%$, while the drug-loading rate of silybin meglumine-loaded solid silica nanoparticles was only $5.78 \% \pm 0.12 \%$. The results fully demonstrate the advantages of empty HMSNs over solid silica nanoparticles in drug delivery.

\section{In vitro release studies}

The in vitro release profiles of silybin meglumine from HMSNs are illustrated in Figure 3, in which silybin meglumine-loaded solid silica nanoparticles were used as a comparison. In order to select a suitable dissolution medium, several kinds of solutions were tested, including artificial
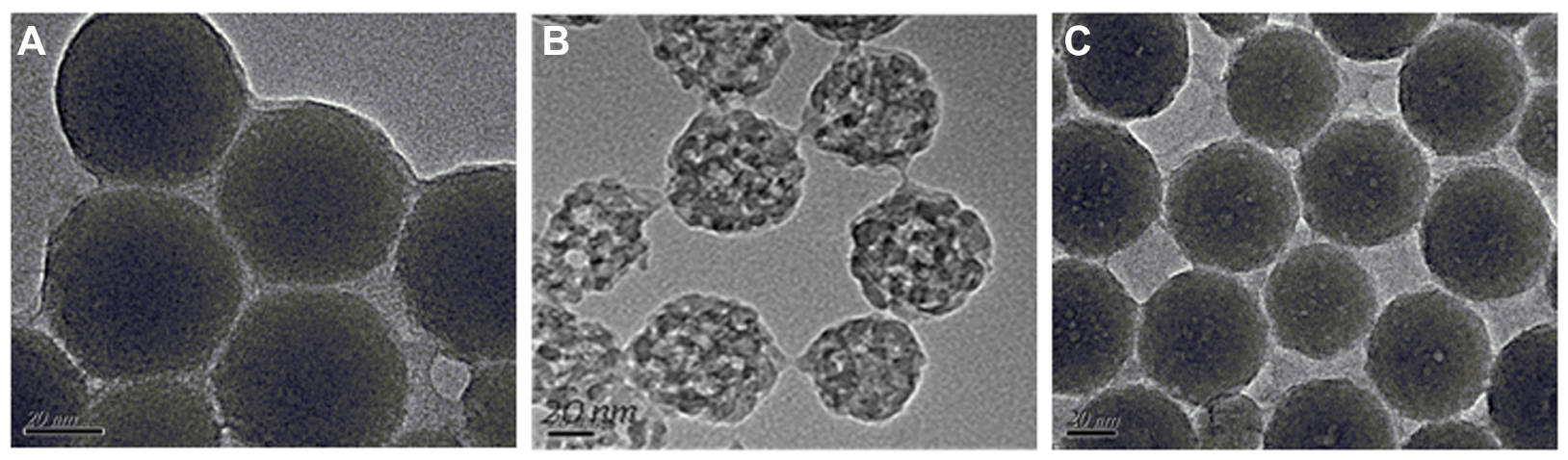

Figure I Images of (A) TEM image for solid silica nanoparticles, (B) TEM image of empty HMSNs, and (C) TEM image of silybin meglumine-loaded HMSNs. Abbreviations: HMSNs, hollow-type mesoporous silica nanoparticles; TEM, transmission electron microscopy. 


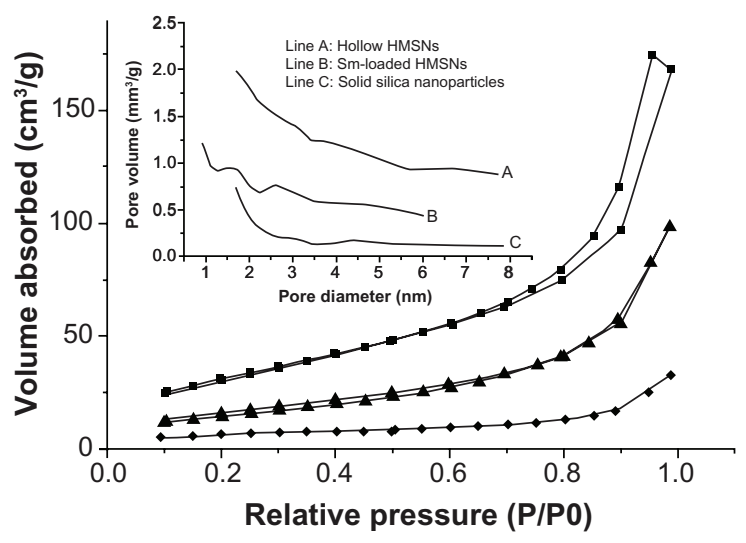

Figure 2 Nitrogen adsorption-desorption isotherms of solid silica nanoparticles ( $\downarrow)$, empty HMSNs ( $(\boldsymbol{\Delta})$, and silybin meglumine-loaded HMSNs (घ). Figure 2 insert: line (A) represents empty HMSNs, line (B) represents silybin meglumine-loaded HMSNs, and line (C) represents solid silica nanoparticles.

Abbreviation: HMSNs, hollow-type mesoporous silica nanoparticles.

gastric juice ( $\mathrm{pH} \mathrm{1.2)} \mathrm{and} \mathrm{artificial} \mathrm{intestinal} \mathrm{juice} \mathrm{(} \mathrm{pH} 6.8$ ), $0.01 \mathrm{M} \mathrm{NaOH}$ solution ( $\mathrm{pH} 12$ ), as well as $\mathrm{Na}_{2} \mathrm{CO}_{3}$ solutions with different concentrations $(0.01 \mathrm{M} \mathrm{pH} 11.1,0.06 \mathrm{M} \mathrm{pH}$ $11.5,0.08 \mathrm{MpH} 11.6$, and $0.1 \mathrm{MpH} 11.7)$. For the preliminary research, artificial gastric and intestinal juices were used to dissolve samples according to the paddle method recorded by the Chinese Pharmacopoeia (2005). As shown in Figure 3A, the accumulated release percentages of silybin meglumin from silybin meglumine-loaded HMSNs in artificial gastric and intestinal juices were less than $20 \%$ within 72 hours. It is obvious that artificial gastric and intestinal juices were not suitable for in vitro dissolution of silybin meglumine-loaded HMSNs, and more investigations were needed in order to find a more appropriate dissolution media. Therefore, $\mathrm{NaOH}$ and $\mathrm{Na}_{2} \mathrm{CO}_{3}$ solutions were introduced and the in vitro release profiles of silybin meglumine-loaded HMSNs are presented in Figure 3B, which show the slowest release rate in a $0.01 \mathrm{M} \mathrm{Na}_{2} \mathrm{CO}_{3}$ solution and the fastest release rate in a $0.1 \mathrm{M} \mathrm{Na}_{2} \mathrm{CO}_{3}$ solution. It is known that silybin meglumine is an ammonium salt which is chemically stable in basic media. An accelerating dissolution rate was observed with increasing $\mathrm{Na}_{2} \mathrm{CO}_{3}$ concentration. Significant enhancement of dissolution was observed in $0.06 \mathrm{M}, 0.08 \mathrm{M}$, and $0.1 \mathrm{M}$ $\mathrm{Na}_{2} \mathrm{CO}_{3}$ solutions as compared with that in artificial gastric or intestinal juices. A similarly rapid release was also observed in $0.06 \mathrm{M}$ and $0.08 \mathrm{M} \mathrm{Na}_{2} \mathrm{CO}_{3}$ solutions, with an accumulative release rate of approximately $59 \%$ and $63 \%$ at 12 hours, respectively, but a significantly faster release
A

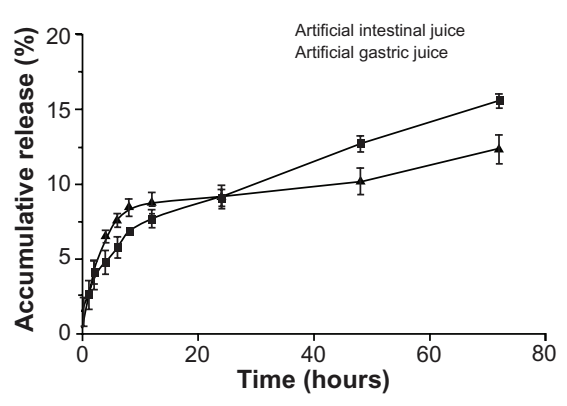

B

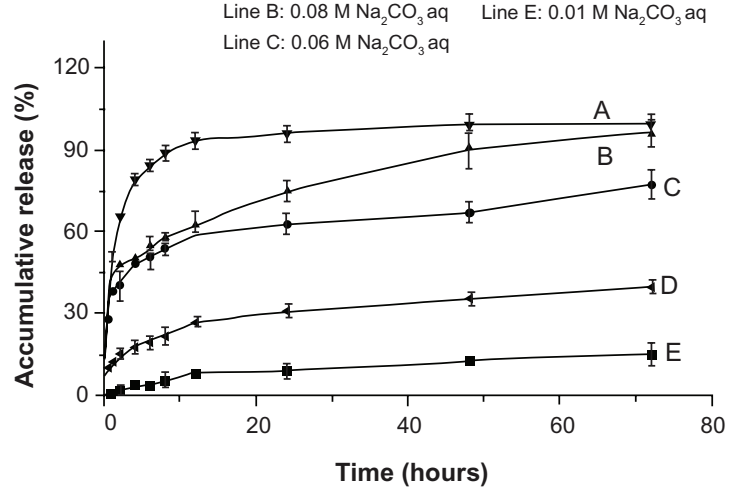

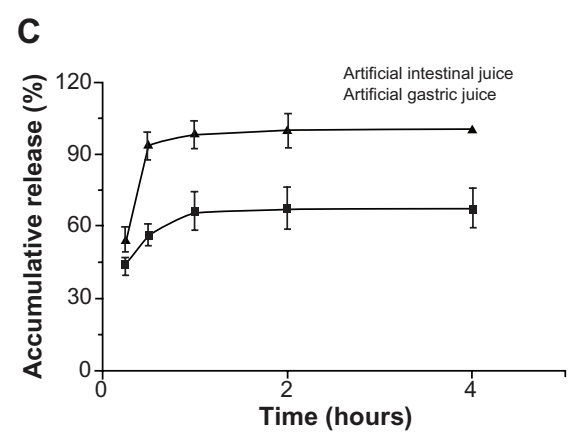

Figure 3 In vitro release curves of silybin from different formulations in various dissolution media. (A) In vitro release curves of silybin meglumine-loaded hollow-type mesoporous silica nanoparticles in artificial gastric juice ( $\mathbf{E})$ and artificial intestinal juice $(\mathbf{A})$; $(\mathbf{B})$ in vitro release curves of silybin meglumine-loaded hollow-type mesoporous silica nanoparticles in $\mathrm{Na}_{2} \mathrm{CO}_{3}$ solutions with different concentrations [0.I M (line A), $0.08 \mathrm{M}$ (line B), $0.06 \mathrm{M}$ (line C), and $0.0 \mathrm{I} \mathrm{M} \mathrm{(line} \mathrm{E)],} \mathrm{and} \mathrm{in} 0.0 \mathrm{I} \mathrm{M} \mathrm{NaOH}$ solution

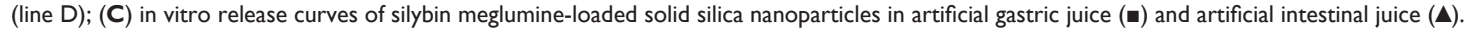

Note: Each datum point represents the mean \pm standard deviation of three administrations. 
from $0.1 \mathrm{M} \mathrm{Na}_{2} \mathrm{CO}_{3}$ solution with an accumulative release rate of approximately $94 \%$ at 12 hours. After that silybin meglumine-loaded HMSNs in a $0.08 \mathrm{M} \mathrm{Na}_{2} \mathrm{CO}_{3}$ solution was faster than that of a $0.06 \mathrm{M} \mathrm{Na}_{2} \mathrm{CO}_{3}$ solution. For $0.08 \mathrm{M} \mathrm{Na}_{2} \mathrm{CO}_{3}$ solution, the accumulative release rate was $80.0 \%$ at 24 hours, followed by a slow increase up to $92.3 \%$ at 72 hours, while the accumulative release rate was only $62.8 \%$ at 24 hours and $77.4 \%$ at 72 hours for $0.06 \mathrm{M}$ $\mathrm{Na}_{2} \mathrm{CO}_{3}$ solution. One of the possible mechanisms for this phenomenon is that silica exhibited weak acidity ${ }^{33,34}$ and $\mathrm{Na}_{2} \mathrm{CO}_{3}$ solution exhibited weak alkalinity, so that an acidbase chemical reaction took place between silica and $\mathrm{Na}_{2} \mathrm{CO}_{3}$, resulting in the water-soluble salt $\mathrm{Na}_{2} \mathrm{SiO}_{3}$ that existed in ion form in water. It could be speculated that the HMSN matrix disintegrated into many microparticles in $\mathrm{Na}_{2} \mathrm{CO}_{3}$ solution, similar to the disintegration of common tablets but at a much slower rate. Furthermore, in $\mathrm{Na}_{2} \mathrm{CO}_{3}$ solutions with different concentrations, there were different degrees of silica dissolution, resulting in different drug-release rates. Therefore, the in vitro release rate of loaded drugs can be controlled by using $\mathrm{Na}_{2} \mathrm{CO}_{3}$ solutions with different concentrations as the dissolution media. In other words, drug release from HMSNs could be affected by the $\mathrm{pH}$ value of the media. It is well known that silica is an acidic oxide that usually reacts with a basic oxide. As a result, silica can dissolve in alkaline media $(\mathrm{pH}>7)$ with stronger alkalinity (a higher $\mathrm{pH}$ value), resulting in a faster reaction. Thus, with the increase in $\mathrm{Na}_{2} \mathrm{CO}_{3}$ concentration, the $\mathrm{pH}$ value of the media increased as well, leading to faster dissolution of HMSNs and quicker release of loaded drugs. However, according to the results (Figure 3B), a slower release profile was seen from $0.01 \mathrm{M} \mathrm{NaOH}$ solution with a $\mathrm{pH}$ value of 12 than from $0.06 \mathrm{M} \mathrm{Na}_{2} \mathrm{CO}_{3}$ solution. Therefore, $\mathrm{pH}$ value was not the only factor that in vitro degradation of HMSNs but also the concentration. The exact reason for the observation is still unknown and further investigation of the dissolution mechanism will be carried out in future works.

Figure 3C shows the in vitro release profiles for silybin meglumine from silybin meglumine-loaded solid silica nanoparticles in artificial gastric and intestinal juices. The accumulated release achieved a constant level at 0.5 hours for solid silica nanoparticles in artificial intestinal juice (with an accumulated release rate of $100 \%$ ) and at one hour for artificial gastric juice (with an accumulated release rate of $70 \%$ ). These results confirm the advantages of HMSNs over solid silica nanoparticles in drug storage and sustained release.

In this study, $\mathrm{Na}_{2} \mathrm{CO}_{3}$ solutions were used as dissolution media for the first time by our research group. It is of great
Table I Mean plasma concentration-time data for silybin meglumine-loaded incorporated into hollow-type silica nanoparticles following a single dose

\begin{tabular}{lll}
\hline $\begin{array}{l}\text { Time points } \\
\text { (hours) }\end{array}$ & $\begin{array}{l}\text { Mean plasma concentration } \\
\text { of silybin }(\mathbf{n g} / \mathbf{m L})\end{array}$ & $\begin{array}{l}\text { Standard } \\
\text { deviation }\end{array}$ \\
\hline 0.25 & 104.03 & 8.28 \\
0.5 & 129.31 & 8.15 \\
1 & 147.31 & 7.11 \\
2 & 180.97 & 4.50 \\
4 & 203.38 & 4.90 \\
6 & 254.55 & 19.47 \\
8 & 283.00 & 12.47 \\
10 & 312.10 & 14.15 \\
12 & 323.90 & 7.03 \\
16 & 321.79 & 11.37 \\
24 & 324.76 & 14.36 \\
36 & 271.03 & 14.93 \\
48 & 253.09 & 7.66 \\
60 & 209.13 & 18.45 \\
72 & 110.17 & 8.29 \\
\hline
\end{tabular}

Note: ${ }^{B}$ Both positive and negative standard deviations were included.

significance for discovering a desirable in vitro dissolution model for drug-loaded HMSNs with long-term release for 72 hours.

\section{In vivo absorption study}

The oral absorption of silybin meglumine from HMSNs was studied in fasting Beagle dogs. The fasting state was selected to exclude the effect of food lipids, which can be confused with the effect of internal lipids. Because silybin meglumine is a compound of silybin and meglumine, it was speculated that the compound could be easily degraded into silybin and meglumine in the in vivo environment. Thus, silybin blood concentration was used as an indicator of silybin meglumine absorption in vivo.

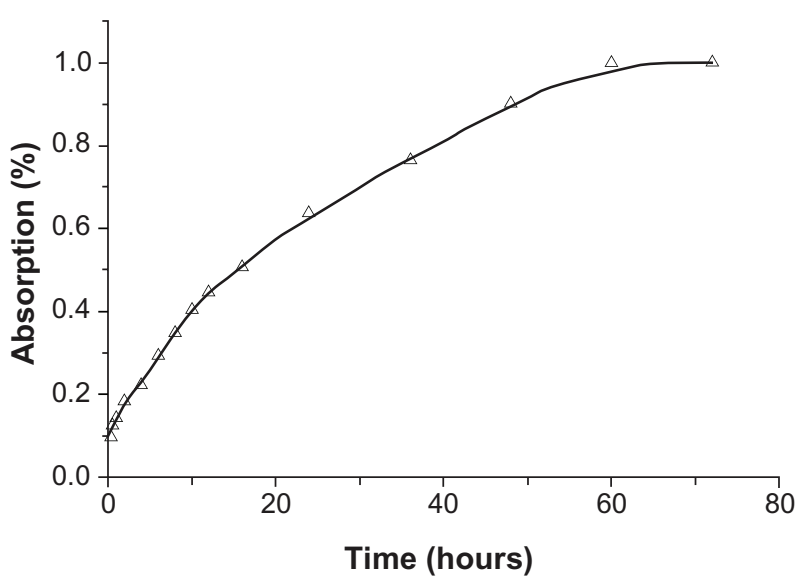

Figure 4 In vivo absorption fraction of silybin meglumine-loaded hollow-type mesoporous silica nanoparticles generated from the Wagner-Nelson method. 
The mean plasma concentration-time data for silybin meglumine-loaded HMSNs following a single dose are presented in Table 1. Based on these data, the percentages of silybin absorbed at specific time points were calculated from the plasma concentration data by using the Wagner-Nelson method, ${ }^{35}$ as shown in Figure 4.

A

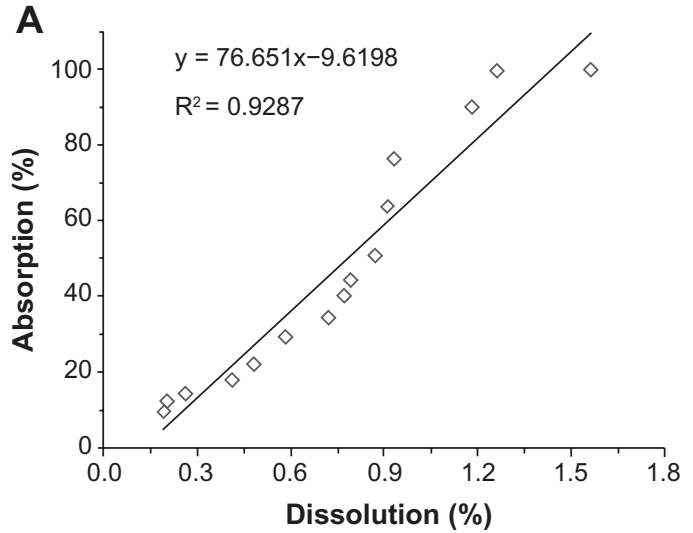

C

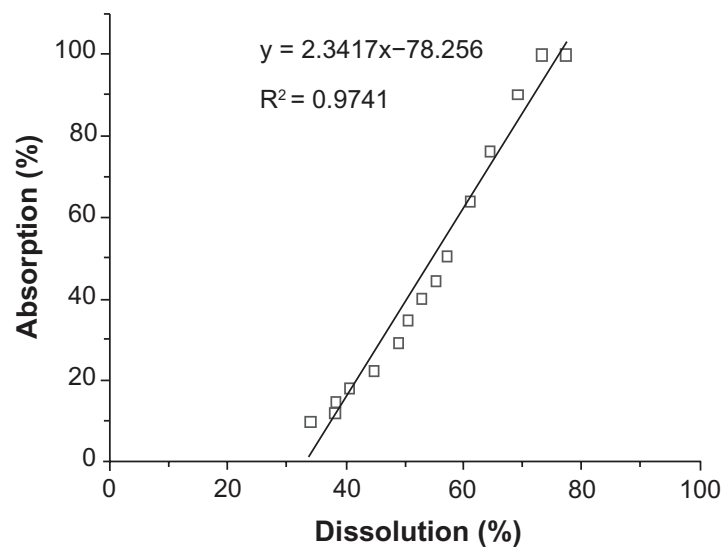

\section{In vitro-in vivo correlation}

The correlations between in vitro and in vivo data for silybin meglumine-loaded HMSNs and silybin meglumine tablets were investigated. Three levels of IVIVC are classified according to the US Food and Drug Administration. ${ }^{36} \mathrm{~A}$ level A correlation, ie, a point-to-point relationship between in

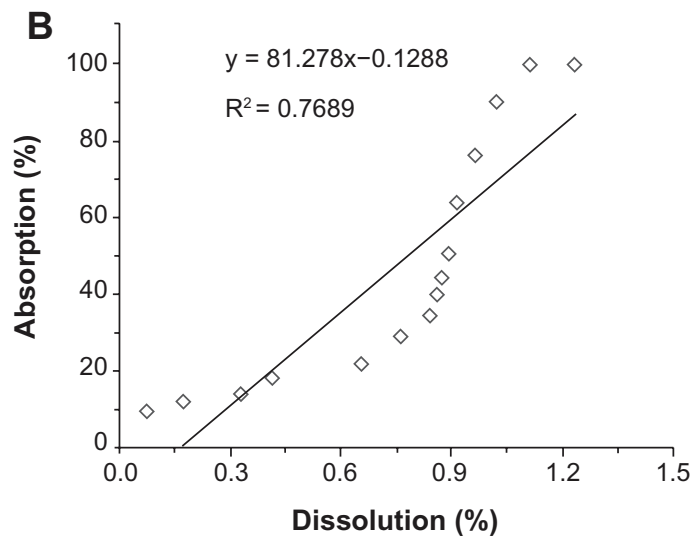

D

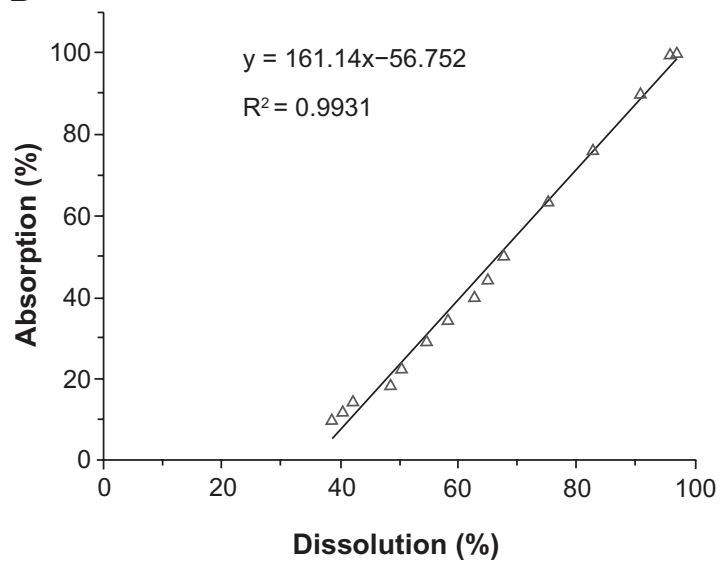

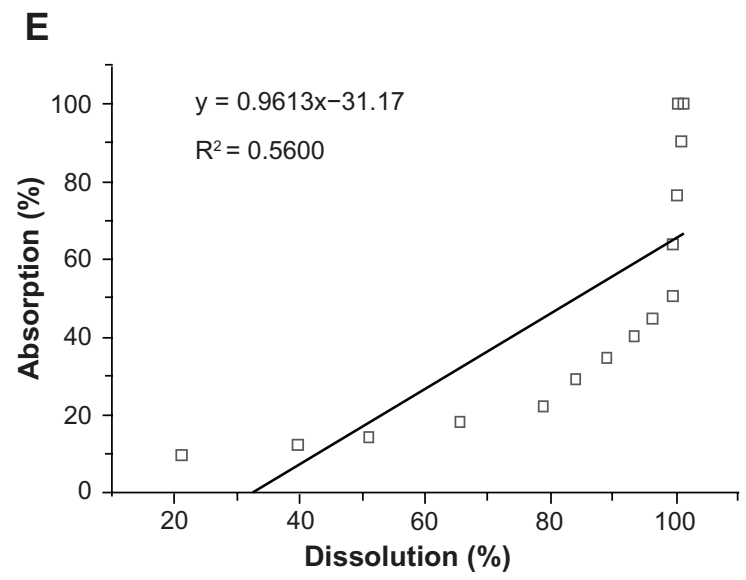

Figure 5 Correlations between in vitro dissolution fractions and in vivo absorption fractions. (A) IVIVC of silybin meglumine-loaded HMSNs in artificial gastric juice; (B) IVIVC of silybin meglumine-loaded HMSNs in artificial intestinal juice; (C) IVIVC of silybin meglumine-loaded $\mathrm{HMSNs}$ in $0.06 \mathrm{M} \mathrm{Na}_{2} \mathrm{CO}_{3}$ solution; (D) IVIVC of silybin meglumine-loaded HMSNs in $0.08 \mathrm{M} \mathrm{Na}_{2} \mathrm{CO}_{3}$ solution; and (E) IVIVC of silybin meglumine-loaded $\mathrm{HMSNs}$ in $0.1 \mathrm{M} \mathrm{Na}_{2} \mathrm{CO}_{3}$ solution.

Abbreviations: HMSNs, hollow-type mesoporous silica nanoparticles; IVIVC, in vitro-in vivo correlations. 
vitro dissolution and in vivo absorption, was used in this present work.

Figure 5 showed the correlations between the in vitro dissolved fractions and in vivo absorbed fractions for silybin meglumine-loaded HMSNs and silybin meglumine tablets in the individual beagle dogs. The fractions dissolved in artificial gastric juice ( $\mathrm{pH}$ 1.2), artificial intestinal juice (pH 6.8), and $0.06 \mathrm{M}, 0.08 \mathrm{M}$, and $0.1 \mathrm{M} \mathrm{Na}_{2} \mathrm{CO}_{3}$ solutions were plotted against the fractions absorbed in 72 hours in the fasting condition, in order to find out the appropriate in vitro dissolution medium leading to better IVIVC. According to the statistics, the linear relationships between the in vitro dissolved fractions and the in vivo absorbed fractions for the silybin meglumine-loaded HMSNs in artificial gastric juice $\left(\mathrm{R}^{2}=0.9287\right.$, as shown in Figure $\left.5 \mathrm{~A}\right)$ and artificial intestinal juice $\left(\mathrm{R}^{2}=0.7689\right.$, as shown in Figure $\left.5 \mathrm{~B}\right)$ were not significantly correlated. In the case of the $0.06 \mathrm{M} \mathrm{Na}_{2} \mathrm{CO}_{3}$ solution as the in vitro dissolution medium, the value of the correlation coefficient $\mathrm{R}^{2}$ was 0.9741 (Figure $5 \mathrm{C}$ ). This value was lower than that for the $0.08 \mathrm{M} \mathrm{Na}_{2} \mathrm{CO}_{3}$ solution, which exhibited a correlation coefficient $\mathrm{R}^{2}$ value of 0.9931 (Figure 5D). Interestingly, when the concentration of $\mathrm{Na}_{2} \mathrm{CO}_{3}$ solution increased to $0.1 \mathrm{M}$, the correlation coefficient $\mathrm{R}^{2}$ decreased dramatically $\left(\mathrm{R}^{2}=0.5600\right)$. This phenomenon indicated that the use of $\mathrm{Na}_{2} \mathrm{CO}_{3}$ solution with concentration lower than 0.08 $\mathrm{M}$ gave a better IVIVC with increasing $\mathrm{Na}_{2} \mathrm{CO}_{3}$ concentration, but when the concentration of $\mathrm{Na}_{2} \mathrm{CO}_{3}$ solution was beyond $0.08 \mathrm{M}$, the correlation coefficient reduced with increasing $\mathrm{Na}_{2} \mathrm{CO}_{3}$ concentration. The exact reasons for this will be addressed in future work. According to the results of IVIVC, the $0.08 \mathrm{M} \mathrm{Na}_{2} \mathrm{CO}_{3}$ solution was the most effective dissolution medium for silybin meglumine-loaded HMSNs. In the case of the $0.08 \mathrm{M} \mathrm{Na}_{2} \mathrm{CO}_{3}$ solution, the in vitro dissolution rate was not the same as the in vivo absorption rate in each subject (Figure 5D). This difference could be due to the differences between the simple in vitro environment and the complex in vivo environment for dissolution. In addition, gastric emptying and intestinal transit would regulate the absorption process, ${ }^{24}$ and therefore result in the differences observed between in vitro dissolution rates and in vivo absorption rates.

In summary, $\mathrm{Na}_{2} \mathrm{CO}_{3}$ solutions had been reported in this study for the first time as an in vitro dissolution medium for drug-loaded HMSNs, which exhibited excellent level A IVIVC. The drug-release model developed in this study achieved in vivo sustained release lasting for 72 hours, with advantages such as fast action, high efficacy, and long-lasting action. The results of this work have therefore had a considerable impact on the construction of sustained-release systems.

\section{Conclusion}

In this study, silybin meglumine was used as the model drug, and was loaded into HMSNs. The silybin meglumine-loaded HMSNs were successfully developed to enhance in vitro dissolution and in vivo absorption, and demonstrated an excellent IVIVC. The results of transmission electron microscopy and nitrogen adsorption showed that the empty HMSNs had a large specific surface area $\left(385.89 \mathrm{~m}^{2} / \mathrm{g}\right)$ and a small pore size (average pore diameter of $2.74 \mathrm{~nm}$ ), which led to a relatively large drug-loading capacity $(58.91 \% \pm 0.39 \%)$. The in vitro dissolution studies conducted in different media revealed that $\mathrm{Na}_{2} \mathrm{CO}_{3}$ solutions of particular concentrations could achieve highly effective, long-acting, sustained release lasting for 72 hours. The results of IVIVC showed that HMSNs in $0.08 \mathrm{M} \mathrm{Na}_{2} \mathrm{CO}_{3}$ solution had a correlation coefficient $\mathrm{R}^{2}$ value of 0.9931 , while the correlation coefficient $\mathrm{R}^{2}$ values for the artificial gastric and intestinal juices were only 0.9287 and 0.7689 , respectively. The IVIVC investigated in this work indicated that HMSNs, together with a $\mathrm{Na}_{2} \mathrm{CO}_{3}$ solution, could achieve an excellent linear relationship between the in vitro dissolution and in vivo absorption for up to 72 hours.

\section{Acknowledgments}

This work was supported by National Natural Science Foundation of China (30472098), Special Funds for 333 Project (BRA2010138), and industry-universityresearch institution cooperation (BY2009141, CY2010023, CZ2009009) in Jiangsu Province and Zhenjiang City. The authors also thank Caleb Kesse Firempong for English editing and the university ethics committee for the kind guidance in the animal experiments.

\section{Disclosure}

The authors report no conflicts of interest in this work.

\section{References}

1. Shi JJ, Votruba AR, Farokhzad OC, Lange R. Nanotechnology in drug delivery and tissue engineering: from discovery to applications. Nano Lett. 2010;10:3223-3230.

2. Murthy SK. Nanoparticles in modern medicine: State of the art and future challenges. Int J Nanomed. 2007;2:129-141.

3. Zhang YZ, Zhi ZZ, Jiang TY, Zhang JH, Wang ZY, Wang SL. Spherical mesoporous silica nanoparticles for loading and release of the poorly water-soluble drug telmisartan. J Control Release. 2010;145: 257-263.

4. Kapoor MP, Vinu A, Fujii W, et al. Self-assembly of mesoporous silicas hollow microspheres via food grade emulsifiers for delivery systems. Micropor Mesopor Mater. 2010;128:187-193.

5. Kilpeläinen M, Riikonen J, Vlasova MA, et al. In vivo delivery of a peptide, ghrelin antagonist, with mesoporous silicon microparticles. J Control Release. 2009;137:166-170. 
6. Slowing II, Vivero-Escoto JL, Wu CW, Lin VSY. Mesoporous silica nanoparticles as controlled release drug delivery and gene transfection carriers. Adv Drug Deliv Rev. 2008;60:1278-1288.

7. Salonen J, Laitinen L, Kaukonen AM, et al. Mesoporous silicon microparticles for oral drug delivery: loading and release of five model drugs. J Control Release. 2005;108:362-374.

8. Vivero-Escoto JL, Slowing II, Trewyn BG, Lin VSY. Mesoporous slica nanoparticles for intracellular controlled drug delivery. Small. 2010;6:1952-1967.

9. Hom C, Lu J, Liong M, et al. Mesoporous silica nanoparticles facilitate delivery of siRNA to shutdown signaling pathway in mammalian cells. Small. 2011;6:1185-1190.

10. Mamaeva V, Rosenholm JM, Bate-Eya LT, et al. Mesoporous silica nanoparticles as drug delivery system for targeted inhibition of notch signaling in cancer. Mol Ther. 2011;19:1538-1546.

11. Rosenholm J, Sahlgren C, Linden M. Cancer-cell targeting and cellspecific delivery by mesoporous silica nanoparticles. J Mater Chem. 2010;20:2707-2713.

12. Chervenkov T, Ivanova D, Galunska B, Gerova D, Yankova T. Toxicity of polymeric nanoparticles with respect to their application as drug carriers. In: Simeonova P, Opopol N, Luster M, editors. NanotechnologyToxicological Issue and Environmental Safety. The Netherlands: Springer; 2007:111-118.

13. Tanaka T, Godin B, Bhavane R, et al. In vivo evaluation of safety of nanoporous silicon carriers following single and multiple dose intravenous administration in mice. Int J Pharm. 2010;402:190-197.

14. Corti G, Cirri M, Maestrelli F, Mennini N, Mura P. Sustained-release matrix tablets of metformin hydrochloride in combination with triacetyl$\beta$-cyclodextrin. Eur J Pharm Biopharm. 2008;68:303-309.

15. Mandal S, Basu SK, Sa B. Sustained release of a water-soluble drug from alginate matrix tablets prepared by wet granulation method. AAPS Pharm Sci Tech. 2009;10:1348-1356.

16. Lee KWY, Nguyen TH, Hanley T, Boyd BJ. Nanostructure of liquid crystalline matrix determines in vitro sustained release and in vivo oral absorption kinetics for hydrophilic model drugs. Int J Pharm. 2009;365:190-199.

17. Chavanpatil MD, Khdair A, Panyam J. Surfactant-polymer nanoparticles: A novel platform for sustained and enhanced cellular delivery of watersoluble molecules. Pharm Res. 2007;24:803-810.

18. Lee TH, Wang J, Wang CH. Double-walled microspheres for the sustained release of a highly water soluble drug: characterization and irradiation studies. J Control Release. 2002;83:437-452.

19. Flora K, Hahn M, Rosen H, Benner K. Milk thistle (Silybum marianum) for the therapy of liver disease. Am J Gastroenterol. 1998;93:139-143.

20. Agarwal R, Katiyar SK, Lundgren DW, Mukhtar H. Inhibitory effect of silymarin, an anti-hepatotoxic flavonoid, on 12-O-tetradecanoylphorbol13-acetate-induced epidermal ornithine decarboxylase activity and mRNA in Sencar mice. Carcinogenesis.1994;15:1099-1103.
21. Ligeret H, Brault A, Vallerand D, Haddad Y, Haddad PS. Antioxidant and mitochondrial protective effects of silibinin in cold preservation-warm reperfusion liver injury. J Ethnopharmacol. 2008;115:507-514.

22. Abenavoli L, Capasso R, Milic N, Capasso F. Milk thistle in liver diseases: past, present, future. Phytother Res. 2010;24:1423-1432.

23. Teng ZG, Han YD, Li J, Yan F, Yang WS. Preparation of hollow mesoporous silica spheres by a sol-gel/emulsion approach. Micropor Mesopor Mater. 2010;127:67-72.

24. Li ZZ, Wen LX, Shao L, Chen JF. Fabrication of porous hollow silica nanoparticles and their applications in drug release control. J Control Release. 2004;98:245-254.

25. Xu W, Gao Q, Xu Y, Wu D, Sun Y. pH-controlled drug release from mesoporous silica tablets coated with hydroxypropyl methylcellulose phthalate. Mater Res Bull. 2009;44:606-612.

26. Li ZZ, Xu SA, Wen LX, et al. Controlled release of avermectin from porous hollow silica nanoparticles: Influence of shell thickness on loading efficiency, UV-shielding property and release. J Control Release. 2006;111:81-88.

27. Wang F, Hui H, Barnes TJ, Barnett C, Prestidge CA. Oxidized mesoporous silicon microparticles for improved oral delivery of poorly soluble drugs. Mol Pharm. 2009;7:227-236.

28. Jinno J, Kamada N, Miyake M, et al. In vitro-in vivo correlation for wet-milled tablet of poorly water-soluble cilostazol. J Control Release. 2008;130:29-37.

29. Wang J, Xiao Q, Zhou HJ, et al. Budded, mesoporous silica hollow spheres: Hierarchical structure controlled by kinetic self-assembly. $A d v$ Mater. 2006;18:3284-3288.

30. Lebedev OI, van Tendeloo G, Collart O, Cool P, Vansant EF. Structure and microstructure of nanoscale mesoporous silica spheres. Solid State Sci. 2004;6:489-498.

31. Yano K, Fukushima Y. Synthesis of mono-dispersed mesoporous silica spheres with highly ordered hexagonal regularity using conventional alkyltrimethylammonium halide as a surfactant. J Mater Chem. 2004;14:1579-1584.

32. Wang JX, Wang ZH, Chen JF, Yun J. Direct encapsulation of watersoluble drug into silica microcapsules for sustained release applications. Mater Res Bull. 2008;43:3374-3381.

33. Damyanova S, Grange P, Delmon B. Surface characterization of zirconia-coated alumina and silica carriers. J Catal. 1997;168: 421-430.

34. Akkari R, Ghorbel A, Esssayem N, Figueras F. Silica supported sulfated zirconia prepared by a sol-gel process: effect of solvent evacuation procedure on the structural, textural and catalytic properties. J Sol Gel Sci Techn. 2006;38:185-190.

35. Akbor MM, Sultana R, Ullah A, Azad MAK, Latif AM, Hasnat A. In vitro-in vivo correlation (IVIVC) of immediate release (IR) levofloxacin tablet. Dhaka Univ J Pharm Sci. 2007;6:113-119.

36. Dressman JB, Reppas C. In vitro-in vivo correlations for lipophilic, poorly water-soluble drugs. Eur J Pharm Sci. 2000;11:S73-S80.
International Journal of Nanomedicine

\section{Publish your work in this journal}

The International Journal of Nanomedicine is an international, peerreviewed journal focusing on the application of nanotechnology in diagnostics, therapeutics, and drug delivery systems throughout the biomedical field. This journal is indexed on PubMed Central, MedLine, CAS, SciSearch $\AA$, Current Contents ${ }^{\circledR} /$ Clinical Medicine,

\section{Dovepress}

Journal Citation Reports/Science Edition, EMBase, Scopus and the Elsevier Bibliographic databases. The manuscript management system is completely online and includes a very quick and fair peer-review system, which is all easy to use. Visit http://www.dovepress.com/ testimonials.php to read real quotes from published authors. 